\title{
A Change in Ocular Circulation after Photocoagulation for Retinopathy of Prematurity in a Neonate
}

\author{
Tadashi Matsumoto $^{a}$ Takashi Itokawa $^{a}$ Tomoaki Shiba $^{a}$ Kotaro Hine $^{b}$ \\ Yuichi Hori ${ }^{\mathrm{a}}$ \\ a Department of Ophthalmology, School of Medicine, Toho University, Tokyo, Japan; \\ ${ }^{b}$ Department of Neonatology, School of Medicine, Toho University, Tokyo, Japan
}

\section{Keywords}

Retinopathy of prematurity $\cdot$ Laser speckle flowgraphy $\cdot$ Mean blur rate $\cdot$ Relative flow volume · LSFG-baby

\begin{abstract}
Background: The dilation of veins and tortuosity of arteries that occur in retinopathy of prematurity (ROP) are improved by therapy, and thus posttherapy changes in ocular circulation are also conceivable. We report a case of ROP in which we were able to measure the ocular circulation before and after photocoagulation therapy, using a laser speckle flowgraphy (LSFG) system that is modified for neonates. Case Report: A female infant was born at 25 weeks' gestation with a birth weight of $808 \mathrm{~g}$. We performed photocoagulation in both eyes at age 15 weeks, and we measured the ocular circulation with the "LSFG-baby" system before and at 12 weeks after the photocoagulation treatment. We also measured the mean blur rate $(\mathrm{MBR})$ in the optic nerve head $(\mathrm{ONH})$ and the relative flow volume (RFV) in the retinal vessels, and we examined the change rates from before to after treatment. The changing rate of the MBR-A (the mean of all values) in the $\mathrm{ONH}$ in the right and left eyes was 76.5 and $98.5 \%$, respectively. The vascular RFV in the severe dilation and tortuosity quadrant also
\end{abstract}




\section{Case Reports in Ophthalmology}

\begin{tabular}{l|l}
\hline Case Rep Ophthalmol 2017:8:91-98 \\
\hline DOI: 10.1159/000456708 & $\begin{array}{l}\text { ( 2017 The Author(s). Published by S. Karger AG, Basel } \\
\text { www.karger.com/cop }\end{array}$ \\
\hline
\end{tabular}

Matsumoto et al.: A Change in Ocular Circulation after Photocoagulation for Retinopathy of Prematurity in a Neonate

tended to decrease. Conclusions: Photocoagulation improved the dilation of veins and tortuosity of arteries and reduced the ocular blood flow in this neonate with ROP. The LSFGbaby system effectively and directly revealed the optic nerve and retinal blood vessels.

(C) 2017 The Author(s)

Published by S. Karger AG, Basel

\section{Introduction}

Retinopathy of prematurity (ROP) is a serious disorder in premature infants. Dilation of veins and tortuosity of arteries are observed in severe ROP, which is improved by treatment such as photocoagulation. It is thus conceivable that impaired ocular circulation is associated with ROP. However, we have been unable to find any reports of the changes in blood flow dynamics from before to after photocoagulation for ROP, and the details of these changes remain unknown.

Laser speckle flowgraphy (LSFG) is based on the changes in the speckle pattern of laser light reflected from the eye, and since LSFG is dependent on the movement of erythrocytes in the retina and choroid [1], it can measure the relative velocity of the erythrocytes' mean blur rate (MBR). LSFG is used to measure the ocular blood flow in various ophthalmic disorders [1-4]. To date, there have been no reports about the use of LSFG as a diagnostic technique for ROP in neonates. This is at least in part because it is difficult to test the ocular blood flow of neonates, as they exhibit high degrees of body and ocular movement and have poor fixation.

We recently established a new system to measure the ocular blood flow in neonates that uses a modified LSFG system, the "LSFG-baby" system [5, 6]. The reproducibility of the LSFGbaby system results was good $[5,6]$. We report herein a case in which we were able to measure the ocular blood flow in an infant with ROP for the first time, and we evaluated the changes in the infant's ocular blood flow before and after photocoagulation.

\section{Case Report}

A female infant born at 25 weeks' gestation with a birth weight of $808 \mathrm{~g}$ was referred to our Pediatric Ophthalmology Department at the age of 32 weeks. Her medical history included necrotizing enterocolitis and patent ductus arteriosus ligation performed at 3 weeks of age.

The ROP had progressed to stage 3, zone 2 with plus disease in the right eye, and stage 2 , zone 2 with pre-plus disease in the left eye at 40 weeks (Fig. 1a, b). Due to a gastrointestinal obstruction surgery planned at 41 weeks of age, the ophthalmological examination and treatment would have been delayed for 1 month. After consulting with the patient's parents, we thus treated both eyes when the patient was 40 weeks old. We performed the photocoagulation for both eyes with the infant under sedation and analgesia using midazolam 0.1 $\mathrm{mg} / \mathrm{kg})$ and fentanyl $(1 \mu \mathrm{g} / \mathrm{kg})$. The conditions of the photocoagulation were: irradiation time $0.3 \mathrm{~s}$, laser power $200 \mathrm{~mW}$, and 591 shots for the right eye and 469 shots for the left eye, using a Novus Spectra Laser System (Lumenis, Tokyo). 


\section{Case Reports in Ophthalmology}

Case Rep Ophthalmol 2017;8:91-98

DOI: $10.1159 / 000456708$

(C) 2017 The Author(s). Published by S. Karger AG, Basel www.karger.com/cop

Matsumoto et al.: A Change in Ocular Circulation after Photocoagulation for Retinopathy of Prematurity in a Neonate

The ophthalmology examination at 3 weeks after treatment revealed that the patient's ROP had improved (Fig. 1c, d). At 12 weeks after the photocoagulation treatment, because the patient was sedated under the same conditions for gastrointestinal postoperative surgical care, we performed an examination for ophthalmology at the same time. In other words, before and at 12 weeks after the photocoagulation treatment, we measured the ocular blood flow primarily in the optic nerve head (ONH) in both eyes using the LSFG-baby system as described [5].

Briefly, testing was performed while the infant was sleeping in order to measure her circulation during rest. Without the use of a lid speculum, the tester gently held the infant's eyelid open with a finger during the examination [5]. The indicators of ocular blood flow that we used in this case were the MBR and the relative flow volume (RFV) [1]. The RFV in the retina is calculated by subtracting the choroidal MBR from the overall MBR. The RFV is thus considered the MBR of only the vascular component.

We measured the MBR in the patient's ONH and the RFV in the retinal vessels by setting a rubber band on the ONH and the vessels (Fig. 2). We used the LSFG Analyzer software (Softcare, Fukuoka, Japan) to analyze the 3 MBR parameters of MBR-A (the mean of all values), MBR-V (the vessel mean), and MBR-T (the tissue mean).

Generally, the ocular perfusion pressure (OPP) is thought to affect the ocular blood flow $[7,8]$, and thus we also measured the following parameters: systolic blood pressure (SBP, $\mathrm{mm} \mathrm{Hg}$ ), diastolic blood pressure (DBP, $\mathrm{mm} \mathrm{Hg}$ ), pulse pressure ( $\mathrm{mm} \mathrm{Hg}$ ), heart rate (beats per min, bpm) measured by a HBP-T105S-N (Omron, Tokyo), and intraocular pressure (IOP, $\mathrm{mm} \mathrm{Hg}$ ) measured by a Tono-Pen Avia (RE Medical, Osaka, Japan). We then calculated the mean arterial blood pressure (MABP, $\mathrm{mm} \mathrm{Hg}$ ) and OPP (mm Hg). The MABP was determined by the formula: DBP + (SBP - DBP $) / 3$. The OPP was defined as (2/3MABP) - IOP.

\section{Results}

The photocoagulation treatment decreased the dilation of veins and the tortuosity of arteries. The respective pretreatment and posttreatment values were as follows: heart rate 124 versus 126 bpm; MABP 57 versus $46 \mathrm{~mm} \mathrm{Hg}$; right-eye IOP 16 versus $13 \mathrm{~mm} \mathrm{Hg}$; lefteye IOP 18 versus $12 \mathrm{~mm}$ Hg; right-eye OPP 22 versus $17 \mathrm{~mm} \mathrm{Hg}$, and left-eye OPP 20 versus $18 \mathrm{~mm}$ Hg. We evaluated all of these parameters after the LSFG measurements were obtained.

The results of the photocoagulation therapy are summarized in Table 1, which indicates decreases in many of the parameters examined. The decreases in the right eye in particular were notable, with MBR-A 23.0, RFV-STRA 444.3, and RFV-STRV 468.6 at pretreatment versus MBR-A 17.6, RFV-STRA 185.3, and RFV-STRV 228.6 at 12 weeks after treatment.

\section{Discussion}

In this patient's case, we measured the ocular circulation of the ROP by using the LSFGbaby system, and we report here for the first time the changes in the ocular circulation before and after photocoagulation. The MBR in the patient's ONH decreased after the photo- 


\section{Case Reports in Ophthalmology}

Case Rep Ophthalmol 2017;8:91-98

DOI: $10.1159 / 000456708$

(C) 2017 The Author(s). Published by S. Karger AG, Basel www.karger.com/cop

Matsumoto et al.: A Change in Ocular Circulation after Photocoagulation for Retinopathy of Prematurity in a Neonate

coagulation for her ROP. The vascular RFV in the severe dilation and tortuosity quadrant also tended to decrease. There were no large differences in heart rate or OPP between before and after the treatment.

Gunay et al. [9] recently reported the changes in blood flow from before to after an intravitreal bevacizumab treatment for ROP; they observed that the blood flow velocity in the ophthalmic artery had decreased. There are differences in treatment methods, but similar results were obtained in this study.

Research groups using LSFG [6] and color Doppler imaging (CDI) $[10,11]$ have reported that the ocular blood flow increases over the course of several weeks after birth. In our patient's case, the blood flow that is believed to increase over several weeks for growth decreased following the photocoagulation for ROP. The decrease in the RFV of the vessels that dominate regions where the ROP was severe was large. As a result, the difference of RFV between the left and right eyes decreased after the treatment.

Based on these results, we believe that in cases in which the surrounding lesions are severe and there is strong dilation of veins and tortuosity of arteries, RFV levels increase, after which changes in veins are corrected with treatment, decreasing RFV. In addition, considering that the ONH MBR decreased greatly in the eye with the more severe condition, i.e. the right eye, we hypothesize that the ONH MBR of this patient was increased due to the severity of ROP at first, and then it was reduced by the photocoagulation treatment.

Several studies have examined the blood flow velocity in the central retinal artery and the ophthalmic artery using CDI [10-13]. Baerts et al. [10] reported that the blood flow velocity in the central retinal artery and the ophthalmic artery did not change based on the onset (or lack thereof) of ROP. However, other studies have reported that the blood flow velocity increased due to ROP onset but decreased due to the manifestation of plus disease $[11,12]$, and yet other studies of plus disease observed no significant differences in blood flow velocity [13]. These inconsistent results may be due to the lack of consideration of the vascular diameter expansion caused by the severity of ROP.

While CDI can measure the absolute value of blood flow speed, it is unable to deal with the changes in blood vessels, making it difficult to measure blood flow volume [10-13]. LSFG can measure the blood flow in the eye directly. While only the relative value is measured, this allows for consideration of the blood flow volume in addition to the speed [1]. However, this method is not as convenient as CDI. Pupillary dilation is required for the measurement, and it must be performed while the patient is asleep or under sedation $[5,6]$.

In our patient's case, photocoagulation decreased the dilation of veins and tortuosity of arteries, and reduced the blood flow. Whether the decreased dilation of veins and tortuosity of arteries was due to decreased blood flow or vice versa remains unclear. The LSFG-baby system used in the present case allowed us to directly visualize the optic nerve and retinal blood vessels. Since the measurement was performed only before and after photocoagulation, it was not possible to investigate changes in blood flow due to the onset of ROP or plus disease.

This brings up the topic of simplification of laboratory techniques and advances in equipment design. However, if we can examine many cases and get a clear idea of how the blood flow changes depending on the treatment, there is a possibility that blood flow measurements may eventually be used in determining the effects of treatments and the patients' prognoses. It is also possible that examining the relation between changes in blood flow and 
changes in pathological conditions may lead to blood-flow measurement becoming a standard procedure when selecting ROP treatment.

Further studies with additional cases are needed to further elucidate the hemodynamics of ROP.

\section{Acknowledgements}

This work was supported by a Toho University project grant (\#26-34), a Toho University Nukada scholarship, and a Toho University Shibata scholarship.

\section{Statement of Ethics}

This study was conducted in accord with the principles laid out in the Declaration of Helsinki. The Ethical Review Committee of Toho University (\#26-96, \#27-19) approved the study. We began this study after a parent of the patient provided informed and written consent.

\section{Disclosure Statement}

The authors have no conflict of interests to declare.

\section{References}

1 Sugiyama T: Basic technology and clinical applications of the updated model of laser speckle flowgraphy to ocular diseases. Photonics 2014;1:220-234.

-2 Sugiyama T, Kojima S, Ishida 0, Ikeda T: Changes in optic nerve head blood flow induced by the combined therapy of latanoprost and beta blockers. Acta Ophthalmol 2009;87:797-800.

-3 Aizawa N, Yokoyama Y, Chiba N, Omodaka K, Yasuda M, Otomo T, Nakamura M, Fuse N, Nakazawa T: Reproducibility of retinal circulation measurements obtained using laser speckle flowgraphy-NAVI in patients with glaucoma. Clin Ophthalmol 2011;5:1171-1176.

4 Shiba T, Takahashi M, Hori Y, Maeno T: Pulse-wave analysis of optic nerve head circulation is significantly correlated with brachial-ankle pulse-wave velocity, carotid intima-media thickness, and age. Graefes Arch Clin Exp Ophthalmol 2012;250:1275-1281.

5 Matsumoto T, Itokawa T, Shiba T, Katayama Y, Arimura T, Mizukaki N, Yoda H, Hori Y: Reproducibility of neonate ocular circulation measurements using laser speckle flowgraphy. Biomed Res Int 2015;693056.

6 Matsumoto T, Itokawa T, Shiba T, Katayama Y, Arimura T, Hine K, Mizukaki N, Yoda H, Hori Y: Ocular blood flow values measured by laser speckle flowgraphy correlate with the postmenstrual age of normal neonates. Graefes Arch Clin Exp Ophthalmol 2016;254:1631-1636.

-7 Geijer C, Bill A: Effects of raised intraocular pressure on retinal, prelaminar, laminar, and retrolaminar optic nerve blood flow in monkeys. Invest Ophthalmol Vis Sci 1979;18:1030-1042.

-8 Tani T, Nagaoka T, Nakabayashi S, Yoshioka T, Yoshida A: Autoregulation of retinal blood flow in response to decreased ocular perfusion pressure in cats: comparison of the effects of increased intraocular pressure and systemic hypotension. Invest Ophthalmol Vis Sci 2014;55:360-367. 
$>9$

Gunay M, Tuten A, Sancak S, Celik G, Bardak H, Dincer E, Karatekin G, Erdogan G, Bardak Y: Effect of single intravitreal bevacizumab on ophthalmic and middle cerebral arterial blood flow in retinopathy of prematurity. Ophthalmic Res 2016;55:165-171.

-10 Baerts W, Wildervanck de Blecourt-Devilee M, Sauer PJ: Ambient light, ophthalmic artery blood flow velocities and retinopathy of prematurity. Acta Paediatr 1993;82:719-722.

11 Hartenstein S, Muller B, Metze B, Czernik C, Buhrer C: Blood flow assessed by color Doppler imaging in retinopathy of prematurity. J Perinatol 2015;35:745-747.

-12 Holland DR, Saunders RA, Kagemann LE, Bluestein EC, Hutchinson AK, Corson DW, Harris A: Color doppler imaging of the central retinal artery in premature infants undergoing examination for retinopathy of prematurity. J AAPOS 1999;3:194-198.

13 Neely D, Harris A, Hynes E, McNulty L, McCranor L, Siesky B, Plager D, Sprunger D, Roberts G: Longitudinal assessment of plus disease in retinopathy of prematurity using color Doppler imaging. J AAPOS 2009;13:509-511.
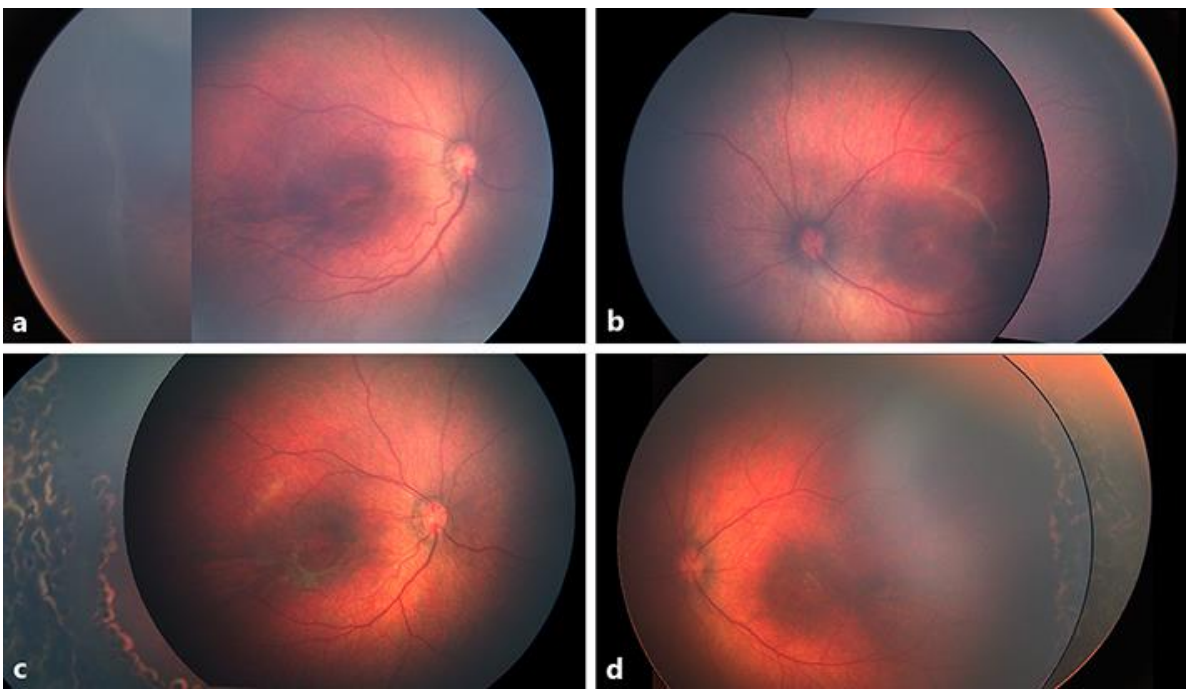

Fig. 1. Fundus photographs (RetCam ${ }^{\circledR}$ ). Photocoagulation improved the dilation of veins and tortuosity of arteries. a Right eye before treatment, stage 3, zone 2 with plus disease. b Left eye before treatment, stage 2 , zone 2 with pre-plus disease. c Right eye at 12 weeks after treatment. $\mathbf{d}$ Left eye at 12 weeks after treatment. 


\section{Case Reports in Ophthalmology}

Case Rep Ophthalmol 2017;8:91-98

(C) 2017 The Author(s). Published by S. Karger AG, Basel www.karger.com/cop

Matsumoto et al: A Change in Ocular Circulation after Photocoagulation for Retinopathy of Prematurity in a Neonate
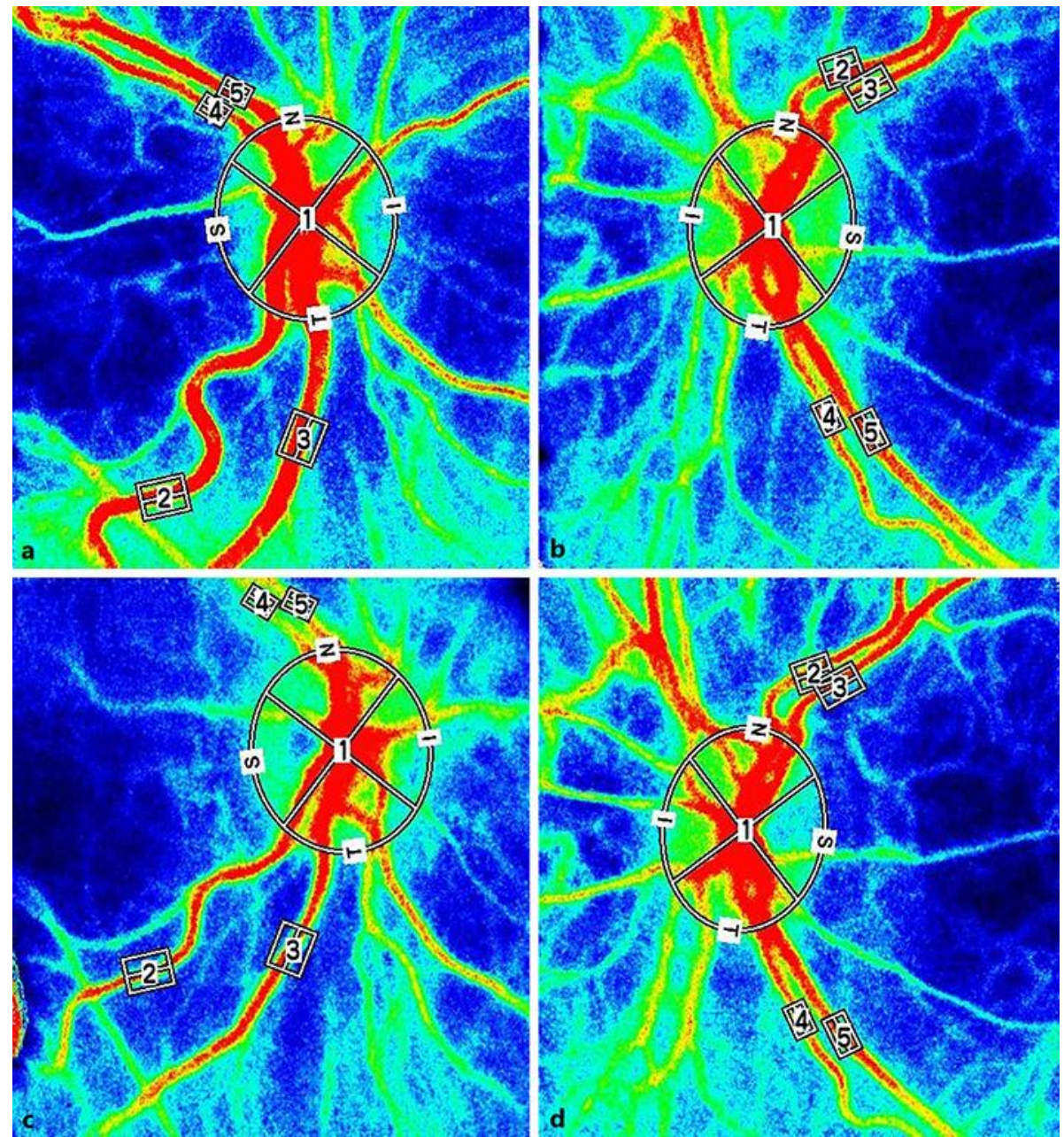

Fig. 2. LSFG images. Red = High blood flow; blue = low blood flow. Photocoagulation improved the dilation of veins and tortuosity of arteries. a Right eye before treatment. b Left eye before treatment. $\mathbf{c}$ Right eye at 12 weeks after treatment. $\mathbf{d}$ Left eye at 12 weeks after treatment. 


\section{Case Reports in Ophthalmology}

\begin{tabular}{l|l}
\hline Case Rep Ophthalmol 2017;8:91-98 \\
\hline DOI: $10.1159 / 000456708$ & $\begin{array}{l}\text { C } 2017 \text { The Author(s). Published by S. Karger AG, Basel } \\
\text { www.karger.com/cop }\end{array}$ \\
\hline
\end{tabular}

Matsumoto et al:: A Change in Ocular Circulation after Photocoagulation for Retinopathy of Prematurity in a Neonate

Table 1. The change rates of the MBR and RFV parameters from before photocoagulation treatment to 12 weeks after treatment in an infant

\begin{tabular}{lllll}
\hline & & $\begin{array}{l}\text { Before } \\
\text { treatment }\end{array}$ & $\begin{array}{l}\text { After } \\
\text { treatment }\end{array}$ & Change rate, \% \\
\hline MBR of $O N H$ & & & & \\
$\mathrm{Rt}$ & MBR-A & 23.0 & 17.6 & 76.5 \\
& MBR-V & 46.7 & 32.6 & 69.8 \\
& MBR-T & 13.8 & 11.8 & 85.5 \\
$\mathrm{Lt}$ & MBR-A & 19.6 & 19.3 & 98.5 \\
& MBR-V & 37.3 & 37.1 & 99.5 \\
& MBR-T & 13.7 & 12.9 & 94.2 \\
\hline$R F V$ & & & & \\
Rt & ITRA & 148.3 & 79.5 & 53.6 \\
& ITRV & 176.7 & 112.9 & 63.9 \\
& STRA & 444.3 & 185.3 & 41.7 \\
Lt & STRV & 468.6 & 228.6 & 48.8 \\
& ITRA & 176.4 & 187.1 & 106.1 \\
& ITRV & 166.3 & 193.3 & 116.2 \\
& STRA & 131.7 & 91.7 & 69.6 \\
& STRV & 228.7 & 173.0 & 75.6 \\
\hline
\end{tabular}

$\mathrm{MBR}$, mean blur rate; $\mathrm{ONH}$, optic nerve head; $\mathrm{RFV}$, relative flow volume; ITRA, inferior temporal retinal artery; ITRV, inferior temporal retinal vein; STRA, superior temporal retinal artery; STRV, superior temporal retinal vein; Rt, right; Lt, left. 